\title{
Serum Elabela/Toddler Levels Are Associated with Albuminuria in Patients with Type 2 Diabetes
}

\author{
Hong Zhang ${ }^{\mathrm{a}}$ Dawei Gong ${ }^{\mathrm{b}}$ Liqiang $\mathrm{Ni}^{\mathrm{c}}$ Lin Shid ${ }^{\mathrm{d}}$ Wendong $\mathrm{Xu}^{\mathrm{a}}$ \\ Min Shi ${ }^{a}$ Juan Chen ${ }^{a}$ Yong $\mathrm{Ai}^{\mathrm{e}}$ Xiangcheng Zhang ${ }^{\mathrm{a}}$
}

aDepartments of Endocrinology and ICU, the Affiliated Huaian No.1 People's Hospital of Nanjing Medical University, Huai'an, China, 'Division of Endocrinology, Diabetes and Nutrition, Department of Medicine, University of Maryland School of Medicine, Baltimore, USA, 'Department of Statistics, University of Central Florida, Orlando, USA, dShanghai Jiaotong University, School of Medicine, Shanghai, China, eDepartment of Pharmaceutical Sciences, School of Pharmacy, University of Maryland, Baltimore, USA

\section{Key Words}

Elabela (ELA) • Diabetic Kidney Disease (DKD) • Albuminuria

\begin{abstract}
Background/Aims: Elabela (ELA) or Toddler is a recently identified hormone that plays a crucial role in embryonic development through the activation of the apelin receptor (APJ). Our previous study indicated that ELA is highly expressed in adult kidney and the ELA receptor signaling pathway is functional in mammalian systems. Whereas nothing is yet known regarding ELA and diabetic kidney disease (DKD). Here, we evaluated the relationship between serum ELA levels and albuminuria in patients with type 2 diabetes (T2D). Methods: An observational study involving 80 patients divided into groups according to their baseline urinary albumin/creatinine ratio (ACR): Group 1 (ACR $\leq 29 \mathrm{mg} / \mathrm{g}$ ), Group 2 (ACR = 30$299 \mathrm{mg} / \mathrm{g}$ ), Group 3 (ACR $\geq 300 \mathrm{mg} / \mathrm{g}$ with normal serum creatinine), and Group 4 (ACR $\geq 300 \mathrm{mg} / \mathrm{g}$ with increased serum creatinine). The demographic, clinical, and biochemical variables including serum ELA were obtained or measured through disease history, physical examination, or laboratory evidence. Results: The results showed that the serum ELA levels decreased gradually with the deterioration of DKD from the stages of normal albuminuria, microalbuminuria, macroalbuminuria, to macroalbuminuria and elevated serum creatinine. In addition, ELA had a significantly negative correlation with ACR $(r=-0.561, P<0.001)$, retinopathy $(r=-0.424, P<0.001)$, serum creatinine $(r=-0.269, P=0.016), \operatorname{SBP}(r=-0.249, P$ $=0.026), \operatorname{DBP}(r=-0.261, P=0.020)$ and a positive correlation with eGFR $(r=0.318, P=0.004)$. Furthermore, stepwise multiple linear regression analysis showed that $A C R$, retinopathy, and LDL-C were considered the most relevant variables to ELA, and ELA, retinopathy, eGFR, and age were important predictors for ACR $(t=-4.546, P=0.000)$. Conclusions: To our knowledge,
\end{abstract}


this is the first study to explore the clinical relationship between ELA levels and CKD. Decreased serum ELA levels might be a significant clinical predictor in patients with DKD or even as a promising agent for treating CKD patients.

(C) 2018 The Author(s)

Published by S. Karger AG, Basel

\section{Introduction}

Diabetic kidney disease (DKD) is now the leading cause of end-stage renal disease (ESRD) $[1,2]$, while the prevalence of diabetes is constantly rising in China and other countries around the world $[3,4]$. The natural course of DKD includes glomerular hyperfiltration, progressive albuminuria, decreasing glomerular filtration rate (GFR), and ultimately ESRD. Metabolic changes associated with hyperglycemia lead to glomerular hypertrophy, glomerulosclerosis, and tubulointerstitial fibrosis [5]. Patients with DKD are at a high risk of cardiorenal morbidity and mortality despite optimum treatment, such as angiotensin-converting enzyme inhibitors and angiotensin receptor blockers, and screening for albuminuria and proteinuria is recommended for risk stratification of these comorbidities [6, 7]. Therefore, the identification of new biomarkers associated with albuminuria and the development of renal-targeted intervention approaches designed to reduce albuminuria are needed to delay the progression of DKD and to decrease cardiovascular disease risk and mortality $[5,8,9]$.

Elabela (ELA), also known as Toddler or Apela, is a peptide of 54 amino acids including a secretory signal with a mature form containing 32 amino acids newly discovered by two research groups $[10,11]$. ELA is an endogenous ligand of the apelin receptor (APJ), a G protein-coupled receptor (GPCR), expressed in multiple tissues including the kidney. It acts on during embryogenesis and circulating in blood in the adult as a secreted hormonal peptide [11]. Our previous study found that ELA is exclusively expressed in human pluripotent stem cells and adult kidney and can activate APJ signaling pathways, induce angiogenesis in umbilical vascular endothelial cells, and relax mouse aortic blood vessels [12]. A recent study indicated that ELA has protective effects on ischemia-reperfusion injury in kidneys and cultured renal cells through anti-inflammatory, antiapoptotic, and antifibrotic actions [13]. Since ELA is predominantly expressed in the kidney, we reasoned that serum ELA levels may change with the severity of kidney damage, and therefore may serve as a functional marker of the kidney in patients with DKD. In this study, we measured serum ELA levels in patients with type 2 diabetes (T2D) with or without albuminuria, and determined the relationship of ELA levels with DKD stage and metabolic indices.

\section{Materials and Methods}

\section{Subjects and examination}

The study was conducted in accordance with the ethical principles stated by the Declaration of Helsinki and with approval from the Ethics Committee of the Affiliated Huaian No.1 People's Hospital of Nanjing Medical University. Informed consent was obtained from all participants. A total of 80 patients with T2D were recruited during the years 2014-2016 for this observational study. The inclusion criteria for the patients were as follows: (1) a diagnosis of T2D according to the 1999 World Health Organization diagnosis and classification criteria; (2) a glycosylated hemoglobin (HbA1c) value of 6.0-13.0\%; (3) a body mass index (BMI) between 18.5 and $28.0 \mathrm{~kg} / \mathrm{m}^{2}$; (4) age $\geq 18$ years; and (5) a baseline urinary albumin/ creatinine ratio (ACR) level after determination using 2 samples. The exclusion criteria comprised patients with a history of disease, physical examination, or laboratory evidence who: (1) had chronic kidney disease with hemodialysis; (2) had primary nephropathy or other secondary kidney disease; (3) had acute diabetic complications; and (4) had liver disease, heart failure, stroke, pregnancy, steroid or nitrate usage, rheumatic disease, or an autoimmune disease.

The patients were divided into 4 groups based on their urinary ACR: Group $1(n=20$, without albuminuria, ACR $\leq 29 \mathrm{mg} / \mathrm{g})$, Group $2(n=20$, with microalbuminuria, ACR $=30-299 \mathrm{mg} / \mathrm{g})$, Group 3 ( $n=20$, with macroalbuminuria and normal serum creatinine, ACR $\geq 300 \mathrm{mg} / \mathrm{g})$, and Group $4(n=20$, 
with macroalbuminuria and increased serum creatinine, ACR $\geq 300 \mathrm{mg} / \mathrm{g}$ ) [14].

Venous blood samples were collected for the measurement of fasting plasma glucose (FPG), HbA1c, blood urine nitrogen (BUN), serum creatinine, total cholesterol (t-CHOL), highdensity lipoprotein cholesterol (HDL-C), low-density lipoprotein cholesterol (LDL-C), triglyceride (TG), and serum ELA. Urine was collected on 2 separate occasions within 7 days, and the average value of both test results was used as the ACR level. Urinary albumin was measured using immunonephelometry (DCA 2000; Bayer, Leverkusen, Germany), and urinary creatinine was determined using the alkaline picrate method. The ACR was calculated as albumin (mg)/creatinine (g). Estimated GFR (eGFR) was calculated using the MDRD equation [15]. Glucose levels were determined by enzymatic methods in plasma samples. HbA1c was measured using high-performance liquid chromatography in EDTA whole blood samples (D10; Bio-Rad, Hercules, CA, USA). Serum ELA levels were determined using an ELABELA (human)-EIA kit (Peninsula Laboratories International, Inc., San Carlos, CA, USA) according to the manufacturer's instructions; this assay has inter- and intra-assay coefficients of variation of less than $8 \%$ and $5 \%$, respectively.

\section{Statistical analysis}

Data are expressed as the mean \pm standard error of the mean (SEM) for normally distributed data or median with 25th and 75th quartiles for skewed data. Data were analyzed by R 3.3.1 (R foundation for Statistical Computing, Vienna, Austria). One-way ANOVA with Bonferroni's correction was applied for comparisons among groups. Multiple linear regression and Pearson correlation analyses were used to assess the relationship among variables. A $P$-value $<0.05$ was considered statistically significant.

\section{Results}

\section{Characteristics of study subjects}

Table 1 presents a statistical summary of the characteristics of all subjects. There were no significant inter-group differences in the following variables: age, BMI, FPG, 2-h PG, HbA1c, diastolic blood pressure (DBP), t-CHOL, LDL-C, HDL-C, and TG. However, Group 1 had a shorter duration of diabetes compared with Group $2(P=0.030)$, Group $3(P=0.043)$, and Group $4(P=0.000)$. The patients in Group 4 had a significantly higher systolic blood

Table 1. The demographic, clinical and biochemical characteristics of all subjects. Note: Data are expressed as the mean \pm SEM or as median (interquartile range). Group 1: diabetic patients without albuminuria; patients with macroalbuminuria and normal serum creatinine; Group
piabetic patients with microalbuminuria; Group 3: diabetic 4: diabetic patients with macroalbuminuria and increased serum HbA1c: glycosylated hemoglobin; SBP: systolic blood pressure; DBP: diastolic blood pressure; t-CHOL: total cholesterol; HDL-C: highdensity lipoprotein cholesterol; LDL-C: low-density lipoprotein creatinine; eGFR: estimated glomerular filtration rate; ACR: urinary albumin/creatinine ratio. The data are expressed as the mean \pm SEM for normally distributed data or median with 25 th and 75 th quartiles for skewed data. ${ }^{\mathrm{a} P}<0.05$ vs. Group 1 ; ${ }^{\mathrm{b}} \mathrm{P}<0.05$ vs. Group 2; ${ }^{\mathrm{c}} \mathrm{P}<0.05$ S. Group 3

\begin{tabular}{lcccc}
\hline Variables & Group 1 & Group 2 & Group 3 & Group 4 \\
\hline Cases (M/F) & $20(10 / 10)$ & $20(10 / 10)$ & $20(14 / 6)$ & $20(13 / 7)$ \\
Age (years) & $55.00 \pm 11.63$ & $61.50 \pm 10.78$ & $54.20 \pm 12.33$ & $60.20 \pm 12.83$ \\
Dur (years) & $6.53 \pm 5.63$ & $10.80 \pm 6.29$ & $10.50 \pm 5.78^{\mathrm{a}}$ & $13.80 \pm 6.64^{\mathrm{a}}$ \\
DR (\%) & 10.0 & $55.0^{\mathrm{a}}$ & $65.0^{\mathrm{a}, \mathrm{b}}$ & $85.0^{\mathrm{a}, \mathrm{b}, \mathrm{c}}$ \\
BMI (kg/m²) & $23.18 \pm 1.20$ & $24.20 \pm 1.59$ & $24.19 \pm 2.06$ & $24.15 \pm 1.52$ \\
FPG (mmol/L) & $10.40 \pm 3.42$ & $8.69 \pm 3.31$ & $10.30 \pm 3.81$ & $8.64 \pm 3.53$ \\
HbA1c (\%) & $8.94 \pm 1.41$ & $8.59 \pm 1.78$ & $9.00 \pm 1.48$ & $8.56 \pm 1.96$ \\
SBP (mmHg) & $133.40 \pm 13.77$ & $135.80 \pm 20.15$ & $144.95 \pm 15.07$ & $159.60 \pm 23.44^{\mathrm{a}, \mathrm{b}, \mathrm{c}}$ \\
DBP (mmHg) & $77.55 \pm 7.52$ & $77.10 \pm 8.02$ & $82.80 \pm 10.58$ & $85.95 \pm 16.31$ \\
t-CHOL (mmol/L) & $4.47 \pm 1.10$ & $4.32 \pm 1.06$ & $4.97 \pm 1.21$ & $4.69 \pm 1.35$ \\
LDL-C (mmol/L) & $2.52 \pm 0.75$ & $2.60 \pm 0.85$ & $2.95 \pm 0.71$ & $2.81 \pm 0.94$ \\
HDL-C (mmol/L) & $1.32 \pm 0.26$ & $1.18 \pm 0.23$ & $1.29 \pm 0.32$ & $1.17 \pm 0.26$ \\
TG (mmol/L) & $1.50 \pm 0.63$ & $1.64 \pm 0.85$ & $2.14 \pm 1.85$ & $1.74 \pm 0.70$ \\
Cre (umol/L) & $52.62 \pm 11.64$ & $61.74 \pm 19.33$ & $70.03 \pm 19.02$ & $200.12 \pm 106.25^{\mathrm{a}, \mathrm{b}, \mathrm{c}}$ \\
BUN (mmol/L) & $5.62 \pm 1.37$ & $6.01 \pm 1.48$ & $6.52 \pm 2.16$ & $13.96 \pm 10.70^{\mathrm{a}, \mathrm{b}, \mathrm{c}}$ \\
eGFR (mL/min/1.73 m²) & $127.41 \pm 22.83$ & $110.28 \pm 37.77$ & $103.40 \pm 35.63$ & $32.13 \pm 12.40^{\mathrm{a}, \mathrm{b}, \mathrm{c}}$ \\
ACR (mg/g) & $6.8(3.4,11.6)$ & $92.3(51.9,133.7)^{\mathrm{a}}$ & $476.7(377.6,773.8)^{\mathrm{a}, \mathrm{b}}$ & $558.1(369.6,1146.6)^{\mathrm{a}, \mathrm{b}}$ \\
ELA (ng/mL) & $9.50 \pm 2.89$ & $7.67 \pm 3.21^{\mathrm{a}}$ & $6.28 \pm 2.85^{\mathrm{a}}$ & $5.26 \pm 2.21^{\mathrm{a}, \mathrm{b}}$ \\
\hline
\end{tabular}




\section{Cellular Physiology Cell Physiol Biochem 2018;48:1347-1354 \begin{tabular}{ll|l} 
DOI: 10.1159/000492093 & $\begin{array}{l}\text { O 2018 The Author(s). Published by S. Karger AG, Basel } \\
\text { www.karger.com/cpb }\end{array}$ \\
\cline { 2 - 3 }
\end{tabular} \\ Zhang et al.: Elabela and Diabetic Kidney Disease}

Fig. 1. Boxplot of ELA levels by group. Group 1: diabetic patients without albuminuria; Group 2: diabetic patients with microalbuminuria; Group 3: diabetic patients with macroalbuminuria and normal serum creatinine; Group 4: diabetic patients with macroalbuminuria and increased serum creatinine. Data are expressed as the mean \pm SEM.

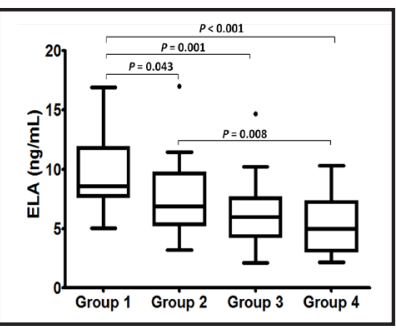

pressure (SBP), but not DBP, compared with the other three groups $(P=$ $0.000, P=0.000$, and $P=$ 0.015 vs. Group 1, 2, and 3, respectively). Concomitant with the levels of serum creatinine and BUN, the patients in Group 4 also had a significantly lower eGFR compared with the other groups $(P=0.000, P=0.000$, and $P=0.000$, vs. Group 1,2 , and 3 , respectively).

\section{ELA levels with different stages of DKD}

Next, we further determined the ELA levels with respect to DKD stage. As shown in Fig. 1, serum ELA levels decreased gradually with the deterioration of DKD from normalalbuminuria (Group 1), microalbuminuria (Group 2), macroalbuminuria with normal serum creatinine (Group 3), to macroalbuminuria with elevated serum creatinine (Group 4). Pairwise comparisons showed significant differences in ELA levels for the comparison of Group 1 with Group 2 $(P=0.043)$, Group $3(P=0.001)$, and Group $4(P<0.001)$ and for Group 2 with Group $4(P=0.008)$, while the other comparisons had $P$-values larger than 0.05 .

\section{Association of ELA with clinical characteristics}

We studied the relationship between serum ELA levels and other variables. In simple correlation analyses, as shown in Fig. 2 (correlation coefficients between variables) and Table 2 ( $P$-values between variables), ELA had a significantly negative correlation with ACR $(r=-0.561, P<0.001)$,

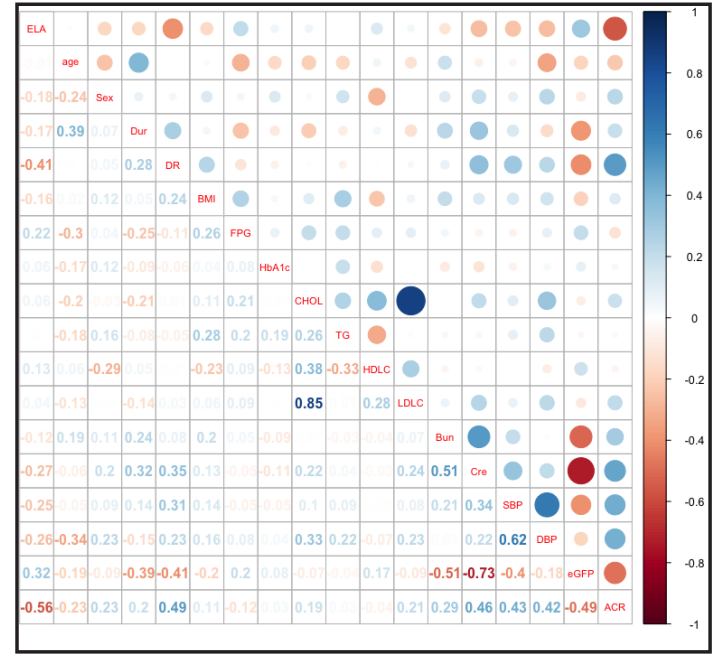

Fig. 2. Correlation coefficients among the characteristics of the study subjects. Orange represents a negative correlation; blue represents a positive correlation. The lower left section of the grid shows the correlation values between the variables, and the upper right section of the grid shows correlation diagrams between the variables. The darker the color and the larger the circle, the larger the correlation coefficient. retinopathy $(r=-0.424, P<0.001)$, serum creatinine $(r=-0.269, P=0.016)$, SBP $(r=-0.249, P=0.026)$, and DBP $(r=-0.261, P=$ $0.020)$ and a positive correlation with eGFR $(r=0.318, P=0.004)$. We also examined the 
relationships among the other variables in Fig. 2 and Table 2. To control for potential confounders, we performed stepwise multiple linear regression with ELA as the response and all of the other aforementioned variables as predictors. We allowed variable selection to proceed in both directions, namely, forward selection and backward elimination. With the Akaike Information Criterion (AIC) as the target, the best model selected was a regression of ELA on retinopathy, LDL-C, and ACR (Table 3). In other words, after taking into account the impact of all variables, retinopathy, LDL-C, and ACR were considered to be the most relevant variables to ELA.

\section{Association of ACR with subject characteristics}

Table 2 shows that ELA, retinopathy, SBP, DBP, eGFR, serum creatinine, and BUN were candidate predictors for the regression of ACR. We then employed stepwise regression analysis in both directions. The optimum model based on the AIC is presented in Table 4, indicating that ELA, age, eGFR, and retinopathy were significant, while DBP and BUN were useful for modelling. The results show that in addition to eGFR, retinopathy, and age, ELA is another significantly relevant variable to ACR.

\section{Discussion}

This study demonstrated that serum ELA might be a new potential predictor in DKD. In the study, serum ELA levels were lowered in patients with albuminuria. More importantly, serum ELA levels decreased progressively with DKD stage from normal-albuminuria, microalbuminuria, macroalbuminuria with normal serum creatinine, to macroalbuminuria and elevated serum creatinine. In addition, serum ELA levels were significantly negatively correlated with ACR, retinopathy, serum creatinine, SBP, and DBP and were positively correlated with eGFR. Moreover, stepwise multiple linear regression analysis indicated that ACR, retinopathy, and LDL-C were the most relevant variables determining ELA levels. We also found that ELA, retinopathy, eGFR, and age were important predictors for ACR by stepwise regression analysis. Thus, ELA appears to be a new biomarker for DKD. However, the sample size of 20 subjects per DKD stage is relatively small, and larger-scale studies are needed to confirm our findings.

ELA is encoded by the $A K 092578$ gene from a region of the human genome that was previously annotated as non-coding RNA. The cDNA encoding ELA is highly conserved in vertebrates, suggesting its essential physiologic functions $[12,16]$. ELA shares functional similarities to apelin, another ligand of the APJ. Apelin, expressed widely in various organs, including the heart, kidney, lung, brain, skeletal muscles, and gastrointestinal tract, is a multifunctional protein involved in angiogenesis, energy metabolism regulation, and fluid homeostasis $[17,18]$. Apelin is reported to have a beneficial effect in diabetes and its complications by promoting glucose uptake, improving insulin sensitivity, reducing body adiposity, enhancing brown adipogenesis, controlling diabetes-induced kidney hypertrophia and albuminuria, and suppressing oxidative stress [19-22]. A clinical study in CKD patients with cardiovascular disease indicated that patients with high serum apelin levels had

Table 3. The stepwise multiple
linear regression analysis of ELA based on the AIC. Note: multiple R-squared: 0.3517 ; F-statistic: 15.28 on 3 and $76 \mathrm{DF}, \mathrm{P}$-value: 7.121e-08. ${ }^{* * *} \mathrm{P}<0.000,{ }^{* *} \mathrm{P}<0.001$, ${ }^{*} \mathrm{P}<0.01,{ }^{*} \mathrm{P}<0.05$

\begin{tabular}{lcccc}
\hline & Estimate & Std Error & $\mathrm{t}$-value & $\mathrm{P}(>|\mathrm{t}|)$ \\
\hline Intercept & 7.7744022 & 1.0765675 & 7.221 & $3.35 \mathrm{e}-10^{+*+}$ \\
& & & & \\
Retinopathy & -1.5200872 & 0.7572464 & -2.007 & $0.0483^{*}$ \\
& & & & \\
LDL-C & 0.6978927 & 0.3620140 & 1.928 & $0.0576^{\#}$ \\
ACR & -0.0036418 & 0.0007646 & -4.763 & $8.95 \mathrm{e}-06^{*+*}$ \\
& & & & \\
\hline
\end{tabular}

Table 4. The stepwise multiple linear regression of ACR based on AIC. Note: multiple R-squared: 0.5448, F-statistic: 16.76 73 DF, P-value: $4.699 \mathrm{e}-12 .{ }^{* * *} \mathrm{P}<$ $0.000,{ }^{* *} \mathrm{P}<0.001,{ }^{*} \mathrm{P}<0.01,{ }^{n} \mathrm{P}<$ 0.05

\begin{tabular}{lcccc}
\hline & Estimate & Std. Error & $\mathrm{t}$-value & $\mathrm{P}(>|\mathrm{t}|)$ \\
\hline Intercep & & & & \\
$\mathrm{t}$ & 763.1881 & 430.4656 & 1.773 & 0.080411 \\
Age & -9.9674 & 3.1314 & -3.183 & $0.002142^{* *}$ \\
DR & 209.0291 & 89.7846 & 2.328 & $0.022680^{*}$ \\
DBP & 6.1910 & 3.2495 & 1.905 & 0.060694 \\
Bun & 8.8764 & 6.0393 & 1.470 & 0.145917 \\
eGFR & -2.3069 & 0.9316 & -2.476 & $0.015591^{*}$ \\
ELA & -46.6379 & 11.8991 & -3.919 & $0.000198^{* * *}$ \\
\hline
\end{tabular}
KARGER 
higher adiponectin levels, greater eGFR, and lower IL-6, resistin, visfatin, and LDL-C levels, with a lower cardiovascular hospitalization and mortality [23]. Conversely, several studies showed opposite results that serum apelin levels had no relationship with diabetes and its vascular complications, and even that apelin promoted diabetic nephropathy by inhibiting proteasomal activity in vitro [24-26]. Thus, the role of apelin in CKD remains controversial.

Chng et al. demonstrated that ELA is expressed earlier than apelin during development and is expressed concomitantly with the APJ before the onset of gastrulation. Since both ELA and apelin act on the APJ, ELA may serve as a biased signaling ligand for the APJ [10]. Yang et al [27]. showed that ELA and apelin were detectable in healthy human plasma at subnanomolar levels, and ELA is found at significantly higher levels than apelin in the circulation. We and others found that ELA transcripts, different from apelin, are expressed mainly in pluripotent stem cells, fetal and adult kidney, prostate, and vascular endothelium, and is predominantly expressed in renal tubular epithelial cells in humans and rodents $[10,12,13,27]$. Our previous study demonstrated that the ELA-APJ signaling pathways are functional in mammalian systems in respect of the suppression of cAMP production, promotion of ERK phosphorylation, induction of angiogenesis, and relaxation of blood vessels. Slowly, the biological functions of ELA are starting to be revealed. Chen et al [13]. reported that ELA treatment markedly improves acute kidney injury in vitro and in vivo through inhibiting apoptosis, reducing inflammatory response, suppressing fibrosis and related markers (TGF- $\beta 1$, fibronectin, vimentin, and collagen 1 ), and decreasing autophagy and the DNA damage response. These data collectively implicate that ELA may play a protective role in kidney diseases including diabetic nephropathy. However, the relationship between serum ELA and DKD had not been characterized prior to the present study.

The present study included 80 diabetic patients at all stages of DKD with comprehensive clinical characteristics. We found a slight decrease of serum ELA levels in patients with microalbuminuria, a further reduction in patients with macroalbuminuria, and an even greater reduction in patients with macroalbuminuria and elevated serum creatinine. Thus, serum ELA levels were progressively reduced with worsening of albuminuria, indicating that the reduction of ELA could be a new predictor for diabetic kidney injury. The reduction of serum ELA may be a result of its decreased synthesis and release from diabetes-inflicted kidney and blood vessel injuries. Intriguingly, serum ELA levels showed a significantly negative correlation with ACR, retinopathy, serum creatinine, SBP, and DBP and a positive correlation with eGFR by pairwise comparisons. Furthermore, stepwise multiple linear regression analysis showed that ACR, retinopathy, and LDL-C were the most relevant variables to ELA level. Conversely, we found that in addition to eGFR, retinopathy, and age, ELA was also a significantly relevant variable to ACR level. Thus, serum ELA levels are related to albuminuria in patients with T2D. In addition, age was included in both linear regressions analyses as a controlling variable, and we found that age was not significant in ELA regression, but it was significant in ACR regression. Since there was no statistically significant difference in age between the 4 groups (Table 1), we consider that age affects ACR levels only as a covariate.

Considering the known functions of ELA in protection against tissue injury, promotion of cell survival, and reduction of blood pressure [10,11, 27-31], it may be reasonable to speculate that the decrease of kidney ELA expression is not just a simple marker for kidney damage but may actually contribute to the DKD symptoms mentioned above. More studies are warranted to delineate the role of ELA in DKD or other kidney injuries.

To the best of our knowledge, this is the first study to explore the clinical relationship between ELA levels and CKD, and showed a significant correlation between ELA levels and the severity of DKD. Nevertheless, this study has some limitations. Firstly, its relatively small sample size might not be representative for the actual relationship between serum ELA levels and overall albuminuria levels in DKD patients. Secondly, healthy subjects were not enrolled, which would limit the observation of ELA levels to diabetic subjects. Further large-scale and longitudinal studies that include healthy subjects would help to clarify the relationship between ELA levels and albuminuria or DKD. Additional experimental and interventional 


\section{Cellular Physiology Cell Physiol Biochem 2018;48:1347-1354 \begin{tabular}{l|l} 
DOI: 10.1159/000492093 & $\begin{array}{l}\text { O } 2018 \text { The Author(s). Published by S. Karger AG, Basel } \\
\text { www.karger.com/cpb }\end{array}$
\end{tabular} \\ Zhang et al.: Elabela and Diabetic Kidney Disease}

studies are also needed to investigate the mechanisms underlying the association of ELA with DKD.

In conclusion, our study shows for the first time that serum ELA levels are reduced in patients with DKD, are significantly associated with albuminuria levels, and are a clinically significant predictor for patients with DKD. If further studies confirm our findings, ELA may serve as a new diagnostic marker or therapeutic agent for CKD.

\section{Acknowledgements}

This study was supported by the National Natural Science Foundation of China Grant Award (81200595/81400807/81700723), the Sixth High-peak Talents Project of Jiangsu Province (WSN-101), Research Project of Jiangsu Provincial 333 Engineering (BRA2016232), Research Project of Jiangsu Provincial Commission of Health and Family Planning (F201549/ H201667), and International Science and Technology Cooperation Project of Huaian (HAC201707).

\section{Disclosure statement}

All authors declare to have no conflict of interests.

\section{References}

1 Zhang L, Long J, Jiang W, Shi Y, He X, Zhou Z, Li Y, Yeung RO, Wang J, Matsushita K, Coresh J, Zhao MH, Wang H: Trends in Chronic Kidney Disease in China. N Engl J Med 2016;375:905-906.

$\longrightarrow 2$ Flyvbjerg A: The role of the complement system in diabetic nephropathy. Nat Rev Nephrol 2017;13:311318.

-3 Wang L, Gao P, Zhang M, Huang Z, Zhang D, Deng Q, Li Y, Zhao Z, Qin X, Jin D, Zhou M, Tang X, Hu Y, Wang L: Prevalence and Ethnic Pattern of Diabetes and Prediabetes in China in 2013. Jama 2017;317:2515-2523.

4 International Diabetes Federation. IDF Diabetes Atlas. 7th. Brussels, Belgium: International Diabetes Federation 2015. http://www.diabetesatlas.org.

5 Alicic RZ, Rooney MT, Tuttle KR: Diabetic Kidney Disease: Challenges, Progress, and Possibilities. Clin J Am Soc Nephrol 2017;12:2032-2045.

6 de Zeeuw D, Bekker P, Henkel E, Hasslacher C, Gouni-Berthold I, Mehling H, Potarca A, Tesar V, Heerspink HJ, Schall TJ: The effect of CCR2 inhibitor CCX140-B on residual albuminuria in patients with type 2 diabetes and nephropathy: a randomised trial. Lancet Diabetes Endocrinol 2015;3:687-696.

7 Velho G, Bouby N, Hadjadj S, Matallah N, Mohammedi K, Fumeron F, Potier L, Bellili-Munoz N, Taveau C, Alhenc-Gelas F, Bankir L, Marre M, Roussel R: Plasma copeptin and renal outcomes in patients with type 2 diabetes and albuminuria. Diabetes Care 2013;36:3639-3645.

-8 Wu J, Shao X, Lu K, Zhou J, Ren M, Xie X, Liu J, Xu Y, Ding Y, Shen X, Zhu C: Urinary RBP and NGAL Levels are Associated with Nephropathy in Patients with Type 2 Diabetes. Cell Physiol Biochem 2017;42:594-602.

-9 Zhang H, Zhang X, Hu C, Lu W: Exenatide reduces urinary transforming growth factor-beta1 and type IV collagen excretion in patients with type 2 diabetes and microalbuminuria. Kidney Blood Press Res 2012;35:483-488.

10 Chng SC, Ho L, Tian J, Reversade B: ELABELA: a hormone essential for heart development signals via the apelin receptor. Dev Cell 2013;27:672-680.

-11 Pauli A, Norris ML, Valen E, Chew GL, Gagnon JA, Zimmerman S, Mitchell A, Ma J, Dubrulle J, Reyon D, Tsai SQ Joung JK, Saghatelian A, Schier AF: Toddler: an embryonic signal that promotes cell movement via Apelin receptors. Science 2014;343:1248636.

12 Wang Z, Yu D, Wang M, Wang Q, Kouznetsova J, Yang R, Qian K, Wu W, Shuldiner A, Sztalryd C, Zou M, Zheng W, Gong DW: Elabela-apelin receptor signaling pathway is functional in mammalian systems. Sci Rep 2015;5:8170. 


\section{Cellular Physiology Cell Physiol Biochem 2018;48:1347-1354 \begin{tabular}{l|l} 
DOI: 10.1159/000492093 & $\begin{array}{l}\text { O } 2018 \text { The Author(s). Published by S. Karger AG, Basel } \\
\text { www.karger.com/cpb }\end{array}$
\end{tabular} \\ Zhang et al.: Elabela and Diabetic Kidney Disease}

13 Chen H, Wang L, Wang W, Cheng C, Zhang Y, Zhou Y, Wang C, Miao X, Wang J, Wang C, Li J, Zheng L, Huang K: ELABELA and an ELABELA Fragment Protect against AKI. J Am Soc Nephrol 2017;28:2694-2707.

14 (9) Microvascular complications and foot care. Diabetes Care 2015;38:S58-66.

15 Fontela PC, Winkelmann ER, Ott JN, Uggeri DP: Estimated glomerular filtration rate in patients with type 2 diabetes mellitus. Rev Assoc Med Bras (1992) 2014;60:531-537.

16 Xie F, Lv D, Chen L: ELABELA: a novel hormone in cardiac development acting as a new endogenous ligand for the APJ receptor. Acta Biochim Biophys Sin (Shanghai) 2014;46:620-622.

17 Chapman NA, Dupre DJ, Rainey JK: The apelin receptor: physiology, pathology, cell signalling, and ligand modulation of a peptide-activated class A GPCR. Biochem Cell Biol 2014;92:431-440.

-18 O'Carroll AM, Lolait SJ, Harris LE, Pope GR: The apelin receptor APJ: journey from an orphan to a multifaceted regulator of homeostasis. J Endocrinol 2013;219:R13-35.

19 Attane C, Foussal C, Le Gonidec S, Benani A, Daviaud D, Wanecq E, Guzman-Ruiz R, Dray C, Bezaire V, Rancoule C, Kuba K, Ruiz-Gayo M, Levade T, Penninger J, Burcelin R, Penicaud L, Valet P, Castan-Laurell I: Apelin treatment increases complete Fatty Acid oxidation, mitochondrial oxidative capacity, and biogenesis in muscle of insulin-resistant mice. Diabetes 2012;61:310-320.

$20 \mathrm{Hu} \mathrm{H}, \mathrm{He} \mathrm{L,} \mathrm{Li} \mathrm{L,} \mathrm{Chen} \mathrm{L:} \mathrm{Apelin/APJ} \mathrm{system} \mathrm{as} \mathrm{a} \mathrm{therapeutic} \mathrm{target} \mathrm{in} \mathrm{diabetes} \mathrm{and} \mathrm{its} \mathrm{complications.} \mathrm{Mol}$ Genet Metab 2016;119:20-27.

21 Day RT, Cavaglieri RC, Feliers D: Apelin retards the progression of diabetic nephropathy. Am J Physiol Renal Physiol 2013;304:F788-800.

22 Zeng H, He X, Hou X, Li L, Chen JX: Apelin gene therapy increases myocardial vascular density and ameliorates diabetic cardiomyopathy via upregulation of sirtuin 3 2014;306:H585-597.

23 Silva AP, Fragoso A, Silva C, Viegas C, Tavares N, Guilherme P, Santos N, Rato F, Camacho A, Cavaco C, Pereira V, Faisca M, Ataide J, Jesus I, Neves P: What is the role of apelin regarding cardiovascular risk and progression of renal disease in type 2 diabetic patients with diabetic nephropathy? Biomed Res Int 2013;2013:247649.

24 Karakoc A, Sahin A, Polat ES, Aliyev E, Yildirim A, Bakan N, Dokumacioglu E: Serum apelin and ADMA levels in type 2 diabetics with and without vascular complications. Diabetes Metab Syndr 2016;10:S106-109.

25 Guo C, Liu Y, Zhao W, Wei S, Zhang X, Wang W, Zeng X: Apelin promotes diabetic nephropathy by inducing podocyte dysfunction via inhibiting proteasome activities. J Cell Mol Med 2015;19:2273-2285.

26 Zhang BH, Wang W, Wang H, Yin J, Zeng XJ: Promoting effects of the adipokine, apelin, on diabetic nephropathy. PLoS One 2013;8:e60457.

27 Yang P, Read C, Kuc RE, Buonincontri G, Southwood M, Torella R, Upton PD, Crosby A, Sawiak SJ, Carpenter TA, Glen RC, Morrell NW, Maguire JJ, Davenport AP: Elabela/Toddler Is an Endogenous Agonist of the Apelin APJ Receptor in the Adult Cardiovascular System, and Exogenous Administration of the Peptide Compensates for the Downregulation of Its Expression in Pulmonary Arterial Hypertension. Circulation 2017;135:1160-1173.

-28 Coquerel D, Chagnon F, Sainsily X, Dumont L, Murza A, Cote J, Dumaine R, Sarret P, Marsault E, Salvail D, Auger-Messier M, Lesur O: ELABELA Improves Cardio-Renal Outcome in Fatal Experimental Septic Shock. Crit Care Med 2017;45:e1139-e1148.

29 Ho L, Tan SY, Wee S, Wu Y, Tan SJ, Ramakrishna NB, Chng SC, Nama S, Szczerbinska I, Chan YS, Avery S, Tsuneyoshi N, Ng HH, Gunaratne J, Dunn NR, Reversade B: ELABELA Is an Endogenous Growth Factor that Sustains hESC Self-Renewal via the PI3K/AKT Pathway. Cell Stem Cell 2015;17:435-447.

30 Ho L, van Dijk M: ELABELA deficiency promotes preeclampsia and cardiovascular malformations in mice. Science 2017;357:707-713.

-31 Schreiber CA, Holditch SJ, Generous A, Ikeda Y: Sustained ELABELA Gene Therapy in High-salt Diet-induced Hypertensive Rats. Curr Gene Ther 2017;16:349-360. 